\title{
ELODIE metallicity-biased search for transiting Hot Jupiters ${ }^{\star}$
}

\section{An intermediate-period Jovian planet orbiting HD 45652}

\author{
N. C. Santos ${ }^{1,2}$, S. Udry ${ }^{2}$, F. Bouchy ${ }^{3}$, R. Da Silva ${ }^{2}$, B. Loeillet ${ }^{3,4}$, M. Mayor ${ }^{2}$, C. Moutou ${ }^{4}$, F. Pont ${ }^{2}$, D. Queloz ${ }^{2}$, \\ S. Zucker ${ }^{5}$ D. Naef ${ }^{2,6}$, F. Pepe ${ }^{2}$, D. Ségransan ${ }^{2}$, I. Boisse ${ }^{3}$, X. Bonfils ${ }^{1,7,8}$, X. Delfosse ${ }^{7}$, M. Desort ${ }^{7}$, T. Forveille ${ }^{7}$, \\ G. Hébrard ${ }^{3}$, A.-M. Lagrange ${ }^{7}$, C. Lovis $^{2}$, C. Perrier ${ }^{7}$, and A. Vidal-Madjar ${ }^{3}$ \\ ${ }^{1}$ Centro de Astrofísica, Universidade do Porto, Rua das Estrelas, 4150-762 Porto, Portugal \\ e-mail: nuno@astro.up.pt \\ 2 Observatoire de Genève, 51 Ch. des Maillettes, 1290 Sauverny, Switzerland \\ 3 Institut d'Astrophysique de Paris, UMR7095 CNRS, Université Pierre \& Marie Curie, 98bis Bd Arago, 75014 Paris, France \\ ${ }^{4}$ Laboratoire d'Astrophysique de Marseille, Traverse du Siphon, 13376 Marseille Cedex 12, France \\ 5 Department of Geophysics and Planetary Sciences, Raymond and Beverly Sackler Faculty of Exact Sciences, Tel Aviv University, \\ Tel Aviv 69978, Israel \\ 6 European Southern Observatory, Casilla 19001, Santiago 19, Chile \\ 7 Laboratoire d'Astrophysique, Observatoire de Grenoble, UJF, CNRS, BP 53, 38041 Grenoble Cedex 9, France \\ ${ }^{8}$ Centro de Astrofísica da Universidade de Lisboa, Observatório Astronómico de Lisboa, Tapada da Ajuda, 1349-018 Lisboa, \\ Portugal
}

Received 15 January 2008 / Accepted 30 April 2008

\section{ABSTRACT}

\begin{abstract}
We present the detection of a $0.47 M_{\text {Jup }}$ planet in a 44-day period eccentric trajectory $(e=0.39)$ orbiting the metal-rich star HD 45652 . This planet, the seventh giant planet discovered in the context of the ELODIE metallicity-biased planet search program, is also confirmed using higher precision radial-velocities obtained with the CORALIE and SOPHIE spectrographs. The orbital period of HD 45652b places it in the middle of the "gap" in the period distribution of extra-solar planets.
\end{abstract}

Key words. stars: individual: HD 45652 - stars: planetary systems - planetary systems: formation - techniques: radial velocities stars: fundamental parameters

\section{Introduction}

More than 280 exoplanets have been found orbiting solar-type stars, most of them discovered using radial-velocity techniques (for a review see Udry \& Santos 2007) ${ }^{1}$. In about 40 cases, the discovered planets are known to transit their host stars. The detection of a photometric signature of a transiting planet, when complemented with its dynamical detection by means of the radial-velocity technique, provides the possibility of deriving its mass, radius, and mean density (e.g. Charbonneau et al. 2000; Pont et al. 2005). These data provide invaluable information about the physical properties of the planet (e.g. its composition - Valencia et al. 2006; Fortney et al. 2007), as well as about its formation and evolution.

It is well known that the probability of finding a giant planet is a strongly rising function of stellar metallicity (Gonzalez 1998; Santos et al. 2004; Fischer \& Valenti 2005). This correlation is generally accepted to reflect the higher probability of forming planets around stars with a higher dust content in the proto-planetary disk, and supports the core-accretion model for

\footnotetext{
* Based on observations collected at the La Silla-Paranal Observatory, ESO (Chile) with the CORALIE spectrograph at the Euler 1.2-m Swiss Telescope, and with the ELODIE and SOPHIE spectrographs at the 1.93-m telescope of the Observatoire de Haute Provence (OHP), France.

${ }^{1}$ See updated tables at http://Www.exoplanets.eu and http://www . exoplanet . eu
}

giant planet formation (e.g. Ida \& Lin 2004b; Benz et al. 2006; Matsuo et al. 2007) $)^{2}$.

Using the metallicity-giant planet relation, several programs are now searching for planets around high metal-content stars (e.g. Tinney et al. 2002; Fischer et al. 2005; Da Silva et al. 2006; Melo et al. 2007). These programs mostly unveiled short-period planets (as expected due to their observing strategy), and increased the number of detected transiting planets orbiting bright stars (e.g. Sato et al. 2005; Bouchy et al. 2005).

One of these programs was based on the former ELODIE spectrograph (Baranne et al. 1996), mounted on the 1.93-m telescope at the OHP observatory (for details of the program see Da Silva et al. 2006). The results of this survey were presented in a series of four papers (Da Silva et al. 2006; Bouchy et al. 2005; Moutou et al. 2006; Da Silva et al. 2007), announcing the discovery of 6 giant planets, one of which transits its star (HD 189733b).

In this paper, we announce the discovery of a $\sim 0.5$ Jupitermass companion orbiting HD 45652, one star from the ELODIE metallicity-biased survey. In Sect. 2, we describe the stellar characteristics of HD 45652. In Sect. 3, we present the radialvelocity measurements used to detect HD 45652b, as well as the derived orbital solution and planetary characteristics. We present our conclusions in Sect. 4, discussing how this new

\footnotetext{
${ }^{2}$ See however recent discussion in Pasquini et al. (2007) and Hekker \& Meléndez (2007).
} 


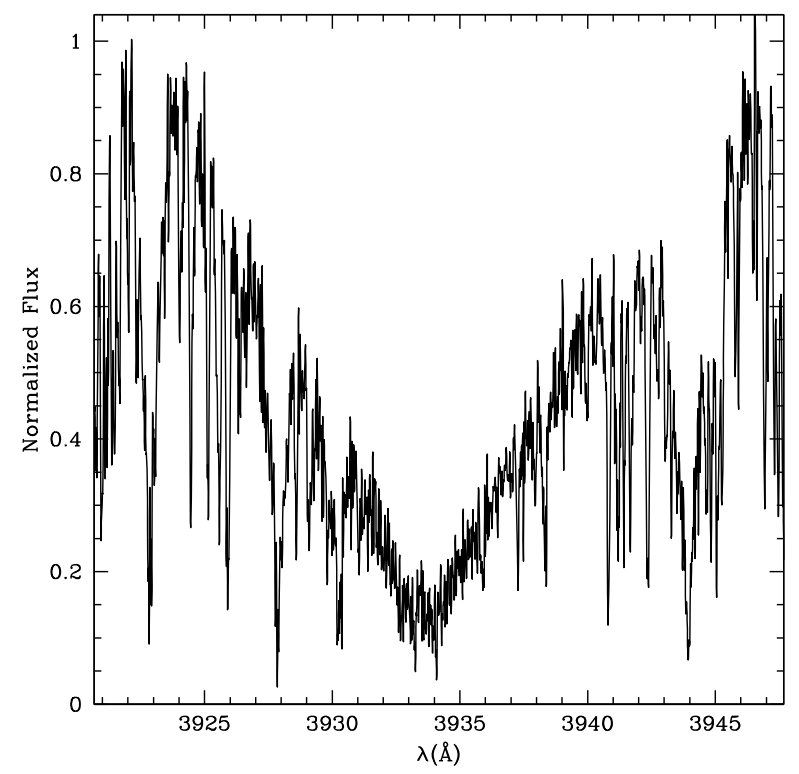

Fig. 1. Spectral region of HD 45652 centered at the Ca II K line (CORALIE spectrum).

intermediate-period planet is placed in the general picture of giant-planet formation models.

\section{Stellar properties of HD 45652}

According to the SIMBAD database, HD 45652 (HIP 30905 , $\mathrm{BD}+111197)$ is a high proper-motion $V=8.1$, $\mathrm{K} 5 \operatorname{star}(B-V=$ 0.85). In the Hipparcos catalogue (ESA 1997), the star is listed as having a parallax of $\pi=27.67 \pm 1.29$ mas, a value that implies a distance of $36 \pm 2 \mathrm{pc}$. No reference for a close companion to HD 45652 is mentioned in this catalogue, and no significant photometric variability was detected.

We used a high-resolution $(R=50000)$ CORALIE spectrum with a signal-to-noise-ratio $(\mathrm{S} / \mathrm{N}) \sim 100$ to derive the stellar parameters and metallicity of HD 45652, using the methodology described in Santos et al. (2004). The resulting effective temperature, surface gravity, and $[\mathrm{Fe} / \mathrm{H}]$ are $5312 \mathrm{~K}, 4.32 \mathrm{dex}$, and +0.29 dex, respectively (see Table 1 ). These parameters are typical of a metal-rich main-sequence late-G or early-K dwarf, and slightly disagree with the spectral type of K5 listed in the Hipparcos catalogue (ESA 1997) and in SIMBAD. The abovementioned temperature is also in good agreement with the value expected on the basis of its $B-V$ colour and metallicity $(5315 \mathrm{~K}$, using the calibration presented in Santos et al. 2004), as well as with other temperature estimates in the literature, namely by Strassmeier et al. $\left(2000, T_{\text {eff }}=5150 \mathrm{~K}\right)$, Allende Prieto \& Lambert $\left(1999, T_{\text {eff }}=5370 \mathrm{~K}\right)$, and Robinson et al. $\left(2007, T_{\text {eff }}=\right.$ $5349 \mathrm{~K})$.

Using stellar evolution tracks from Schaerer et al. (1993), we derived a stellar mass of $0.83 \pm 0.05 M_{\odot}$, and an age above 12 Gyr for HD 45652. Strassmeier et al. (2000) classified this star as chromospherically non-active, in agreement with its derived age. From SOPHIE spectra, we also derived a value of $\log R_{\mathrm{HK}}^{\prime}=-4.90 \pm 0.10$. The low activity level is confirmed by an inspection of the center of the $\mathrm{Ca}$ II $\mathrm{H}$ and $\mathrm{K}$ line regions (see Fig. 1). Such a low level of activity is also compatible with the low value of projected rotational velocity $\left(v \sin i<2 \mathrm{~km} \mathrm{~s}^{-1}\right)$ as derived from the Cross-Correlation Function (CCF) of the ELODIE spectra (Table 1).
Table 1. Stellar parameters for HD 45652.

\begin{tabular}{lcc}
\hline \hline Parameter & Value & Reference \\
\hline Spectral type & K5/G8-K0 & Hipparcos/This Paper \\
$m_{v}$ & 8.1 & Hipparcos \\
$B-V$ & 0.85 & Hipparcos \\
Distance [pc] & $36 \pm 2$ & Hipparcos \\
$v \sin i\left[\mathrm{~km} \mathrm{~s}^{-1}\right]$ & $<2 \dagger$ & This paper \\
$\log R_{\mathrm{HK}}^{\prime}$ & $-4.90 \pm 0.10 \dagger \dagger$ & This paper \\
$T_{\mathrm{eff}}[\mathrm{K}]$ & $5312 \pm 68$ & This paper \\
$\log g[\mathrm{cgi}]$ & $4.32 \pm 0.21$ & This paper \\
$\xi_{\mathrm{t}}$ & $0.89 \pm 0.09$ & This paper \\
{$[\mathrm{Fe} / \mathrm{H}]$} & $+0.29 \pm 0.07$ & This paper \\
$\mathrm{Mass}\left[M_{\odot}\right]$ & $0.83 \pm 0.05$ & This paper \\
\hline
\end{tabular}

$\dagger$ From ELODIE spectra using a calibration similar to that presented by Santos et al. (2002).

$\dagger \dagger$ From SOPHIE spectra using a calibration similar to that presented by Santos et al. (2000).

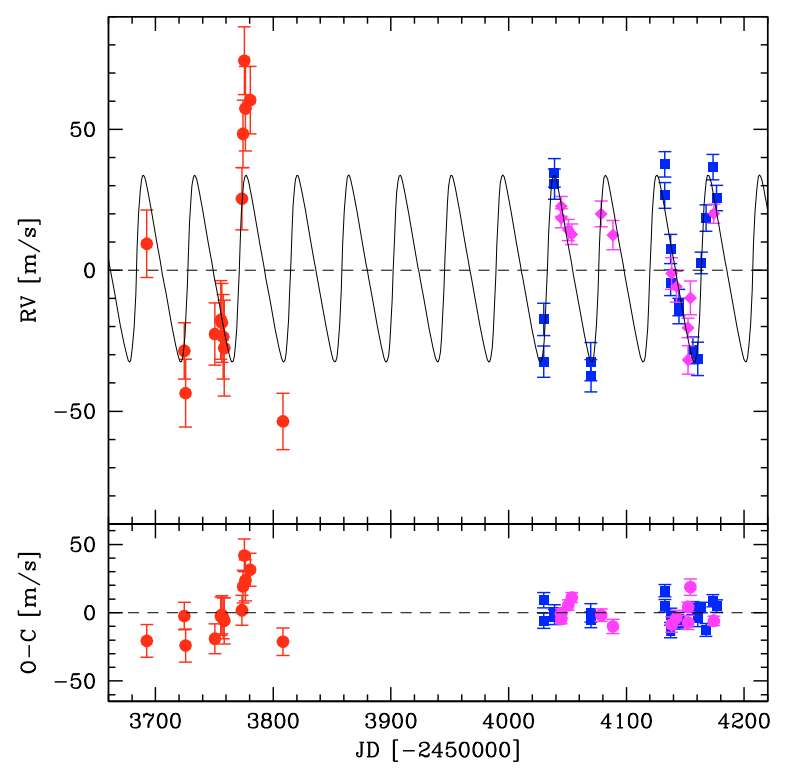

Fig. 2. Top: ELODIE (red circles), CORALIE (blue squares), and SOPHIE (magenta diamonds) radial-velocities for HD 45652 as a function of time, and the best-fit Keplerian function to the data. Bottom: residuals of the fit.

\section{A giant planet orbiting HD 45652}

HD 45652 was part of the ELODIE metallicity-biased planet search sample (Da Silva et al. 2006). A series of 14 radial velocities were obtained with this instrument between October 2005 and March 2006 (Table 3), using the 1.93-m telescope at the Observatoire de Haute Provence (France). The data showed the presence of a clear radial-velocity variation, although the time coverage of the data did not allow us to confirm the nature of the observed signal. The average photon-noise error of the measurements is $12.6 \mathrm{~m} \mathrm{~s}^{-1}$.

HD 45652 was then monitored using the CORALIE spectrograph, at the Euler 1.2-m Swiss telescope (La Silla observatory, ESO, Chile - between October 2006 and March 2007), and with the SOPHIE spectrograph, at the 1.93-m OHP telescope (France - between November 2006 and March 2007). The average photon-noise error of the 18 CORALIE and 12 SOPHIE radial-velocities is $5.5 \mathrm{~m} \mathrm{~s}^{-1}$ and $4.4 \mathrm{~m} \mathrm{~s}^{-1}$, respectively. Both sets of higher quality radial-velocities obtained (Table 3 ) confirm our earlier results, showing a clear radial-velocity signal (Fig. 2). 


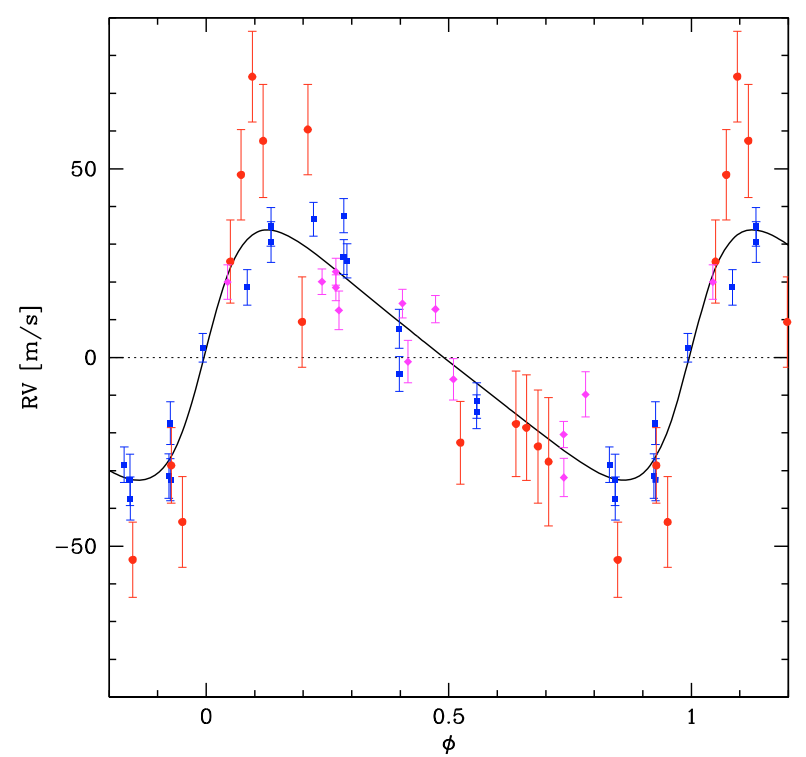

Fig. 3. Phase-folded ELODIE, CORALIE, and SOPHIE radial velocities for HD 45652 at a period of 43.6 days. Symbols are as in Fig. 2.

Table 2. Elements of the fitted orbit for HD $45652 \mathrm{~b}$, derived using the ELODIE, CORALIE, and SOPHIE data.

\begin{tabular}{lll}
\hline \hline$P$ & $43.6 \pm 0.2$ & {$[\mathrm{~d}]$} \\
$T$ & $2454120.3 \pm 1.2$ & {$[\mathrm{~d}]$} \\
$a$ & 0.23 & {$[\mathrm{AU}]$} \\
$e$ & $0.38 \pm 0.06$ & \\
$V_{r}$ (ELODIE) & $-5.117 \pm 0.006$ & {$\left[\mathrm{~km} \mathrm{~s}^{-1}\right]$} \\
$V_{r}$ (CORALIE) & $-5.044 \pm 0.002$ & {$\left[\mathrm{~km} \mathrm{~s}^{-1}\right]$} \\
$V_{r}$ (SOPHIE) & $-4.999 \pm 0.002$ & {$\left[\mathrm{~km} \mathrm{~s}^{-1}\right]$} \\
$\omega$ & $273 \pm 12$ & {$[\mathrm{degr}]$} \\
$K_{1}$ & $33.1 \pm 2.5$ & {$\left[\mathrm{~m} \mathrm{~s}^{-1}\right]$} \\
$\sigma(\mathrm{O}-\mathrm{C})$ & 8.9 & {$\left[\mathrm{~m} \mathrm{~s}^{-1}\right]$} \\
$N$ & 44 & \\
$m_{2} \sin i$ & 0.47 & {$\left[M_{\text {Jup }}\right]$} \\
\hline
\end{tabular}

The combined ELODIE, CORALIE, and SOPHIE radialvelocity measurements are best fited using a Keplerian function with a semi-amplitude $K=33 \mathrm{~m} \mathrm{~s}^{-1}$, an eccentricity $e=0.38$, and a period $P=43.6$ days (see Figs. 2 and 3, and Table 2). The residuals around the fit $\left(8.9 \mathrm{~m} \mathrm{~s}^{-1}\right)$ are within the expected value given the photon-noise errors of the data. Monte Carlo simulation results shows that the false-alarm probability is only $0.1 \%$. The observed Keplerian fit corresponds to the expected radialvelocity variation induced by the presence of a giant planet with a minimum mass of $0.47 M_{\text {Jup }}$, orbiting HD 45652 in an eccentric orbit with a semi-major axis of $0.23 \mathrm{AU}$.

A similar orbital solution is obtained if we use only the CORALIE and SOPHIE data to derive the best-fit Keplerian function (similar orbital parameters are obtained, within the errors). The observed residuals are smaller in this case $\left(7.4 \mathrm{~m} \mathrm{~s}^{-1}\right)$.

A look at the residuals of the Keplerian function fitting (Fig. 2) suggests that the adopted orbital solution does not well describe the ELODIE data. The observed residuals are probably due to the higher quality CORALIE and SOPHIE measurements which are then weighted more signiticantly than the ELODIE measurements when fitting the data. Since we do not have any overlap between the ELODIE and CORALIE datasets, it may be that the zero point of radial-velocity measurements for the different instruments is not well constrained. Alternatively, a second signal could be present in the data. A scrutiny of old CORAVEL
Table 3. ELODIE and CORALIE radial-velocity measurements of HD 45652.

\begin{tabular}{ccc}
\hline \hline JD & $V_{r}\left[\mathrm{~km} \mathrm{~s}^{-1}\right]$ & $\sigma\left(V_{r}\right)\left[\mathrm{km} \mathrm{s}^{-1}\right]$ \\
\hline ELODIE & & \\
2453692.65990 & -5.108 & 0.012 \\
2453724.51830 & -5.146 & 0.010 \\
2453725.52840 & -5.161 & 0.012 \\
2453750.52140 & -5.140 & 0.011 \\
2453755.52560 & -5.135 & 0.014 \\
2453756.46230 & -5.136 & 0.014 \\
2453757.49900 & -5.141 & 0.015 \\
2453758.44960 & -5.145 & 0.017 \\
2453773.45970 & -5.092 & 0.011 \\
2453774.42840 & -5.069 & 0.012 \\
2453775.42810 & -5.043 & 0.012 \\
2453776.41300 & -5.060 & 0.015 \\
2453780.42370 & -5.057 & 0.012 \\
2453808.29880 & -5.171 & 0.010 \\
CORALIE & & \\
2454029.79264 & -5.0607 & 0.0063 \\
2454029.80422 & -5.0757 & 0.0063 \\
2454038.83579 & -5.0093 & 0.0057 \\
2454038.84718 & -5.0125 & 0.0060 \\
2454069.78922 & -5.0759 & 0.0076 \\
2454069.81454 & -5.0807 & 0.0063 \\
2454132.62897 & -5.0005 & 0.0050 \\
2454132.64037 & -5.0107 & 0.0050 \\
2454137.62078 & -5.0233 & 0.0056 \\
2454137.63218 & -5.0301 & 0.0050 \\
2454144.58745 & -5.0442 & 0.0049 \\
2454144.59885 & -5.0368 & 0.0052 \\
2454151.59608 & -5.0445 & 0.0048 \\
2454156.52101 & -5.0726 & 0.0052 \\
2454160.52499 & -5.0747 & 0.0066 \\
2454163.57158 & -5.0404 & 0.0044 \\
2454167.58467 & -5.0250 & 0.0053 \\
2454173.53990 & -5.0061 & 0.0050 \\
2454176.55337 & -5.0187 & 0.0052 \\
SOPHIE & & \\
2454044.67360 & -4.9763 & 0.0035 \\
2454044.68380 & -4.9805 & 0.0034 \\
2454050.66630 & -4.9847 & 0.0038 \\
2454053.63090 & -4.9862 & 0.0036 \\
2454078.56860 & -4.9790 & 0.0045 \\
2454088.56780 & -4.9865 & 0.0051 \\
2454138.40400 & -5.0001 & 0.0056 \\
2454142.48580 & -5.0048 & 0.0055 \\
2454152.40650 & -5.0194 & 0.0034 \\
2454152.41540 & -5.0308 & 0.0051 \\
2454154.37280 & -5.0088 & 0.0059 \\
2454174.29350 & -4.9789 & 0.0033 \\
\hline & &
\end{tabular}

data, which has a precision of $\sim 300 \mathrm{~m} \mathrm{~s}^{-1}$, does not uncover the existence of any long-term radial-velocity trend. We also studied the residuals of the Keplerian fit for the presence of a second significant low-amplitude signal in the data, without success. We thus do not endorse the idea that a second signal is present.

Given the low projected rotational velocity and activity level of the star, we do not expect that activity-related phenomena could induce the observed periodic radial-velocity signal. Although not a fully effective diagnostic for stars rotating very slowly (Santos et al. 2003; Desort et al. 2007), an analysis of the CCF bisectors also lends support to this idea (Fig. 4). No significant correlation is found between the observed radial-velocity and the shape of the CCF denoted by a measurement of the Bisector Inverse Slope, BIS (defined as in Queloz et al. 2001). 


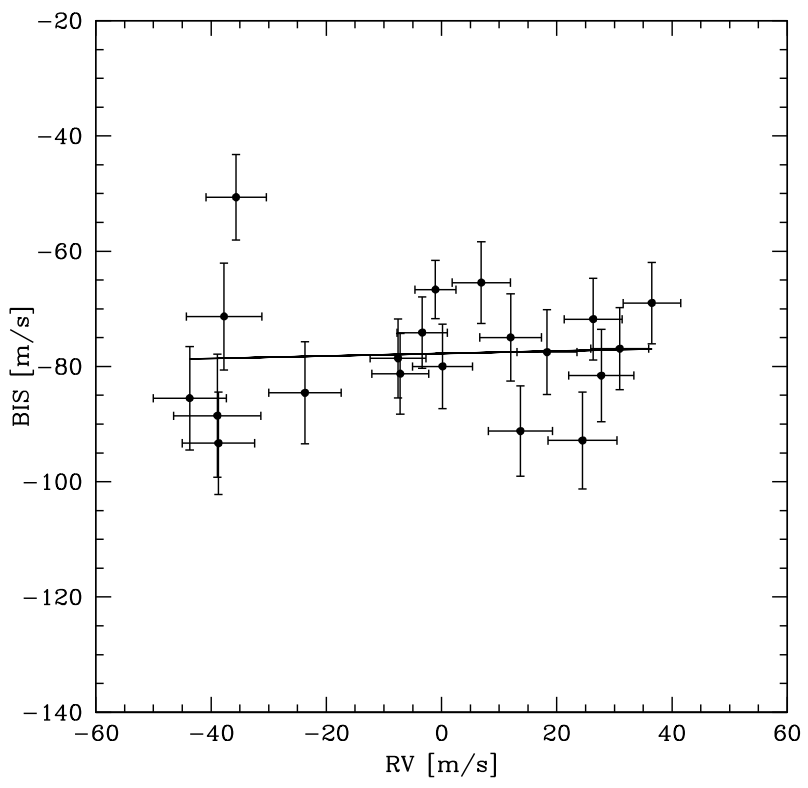

Fig. 4. BIS versus radial-velocity for the CORALIE data of HD 45652. To demonstrate the nonexistence of a correlation, the vertical and horizontal scales were set to be identical. The best linear fit to the data is also shown. The slope of the fit has a non significant value of $0.02 \pm 0.09$.

The observed radial-velocity variation of HD 45652 is therefore best explained by the presence of a planetary-mass companion in a $\sim 44$-day period orbit.

\section{Discussion and concluding remarks}

Statistical studies of the properties of observed extra-solar planets have shown that the orbital period distribution is characterized by a well-defined peak for $P<10$-days, followed by some sort of period valley for values of $P$ up to $\sim 100$ days. Above this latter value, the distribution is again an increasing function of orbital period (Cumming et al. 1999; Udry et al. 2003; Udry \& Santos 2007). The observed shape of the period distribution is predicted by some models of planet formation and evolution (Ida \& Lin 2004a; Mordasini et al. 2007).

With an orbital period of $\sim 44$-days, HD $45652 \mathrm{~b}$ is placed in the middle of the so-called period valley.

Interestingly, Burkert \& Ida (2007) pointed out that the observed period valley is more pronounced for planets orbiting more massive stars (F-dwarfs with mass above $\sim 1.2 M_{\odot}$ ). Furthermore, the period gap appears to be more significant for the higher mass planets $\left(>0.8 M_{\text {Jup }}\right)$. Burkert \& Ida (2007) attributed this observation to shorter timescales of disk depletion for higher-mass stars.
HD $45652 b$, a $0.47 M_{\text {Jup }}$ planet with an orbital period of $\sim 44$ days orbiting a $0.83 M_{\odot}$ star perfectly fits this scenario.

Acknowledgements. We thank the Swiss National Research Foundation (FNRS) and the Geneva University for their continuous support to our planet search programmes. N.C.S. would like to thank the support from Fundação para a Ciência e a Tecnologia, Portugal, in the form of a grant (references POCI/CTEAST/56453/2004 and PPCDT/CTE-AST/56453/2004), and through programme Ciência 2007 (C2007-CAUP-FCT/136/2006). The support from Coordenação de Aperfeiçoamento de Pessoal de Nível Superior (CAPES - Brazil) to R.D.S. in the form of a scholarship is gratefully acknowledged as well. We acknowledge support from the French National Research Agency (ANR) through project grant NT05-4_44463.

\section{References}

Allende Prieto, C., \& Lambert, D. L. 1999, A\&A, 352, 555

Baranne, A., Queloz, D., Mayor, M., et al. 1996, A\&AS, 119, 373

Benz, W., Mordasini, C., Alibert, Y., \& Naef, D. 2006, in Tenth Anniversary of

51 Peg-b: Status of and prospects for hot Jupiter studies, ed. L. Arnold, F.

Bouchy, \& C. Moutou, 24

Bouchy, F., Udry, S., Mayor, M., et al. 2005, A\&A, 444, L15

Burkert, A., \& Ida, S. 2007, ApJ, 660, 845

Charbonneau, D., Brown, T., Latham, D., \& Mayor, M. 2000, ApJ, 529, L45

Cumming, A., Marcy, G. W., \& Butler, R. P. 1999, ApJ, 526, 890

Da Silva, R., Udry, S., Bouchy, F., et al. 2006, A\&A, 446, 717

Da Silva, R., Udry, S., Bouchy, F., et al. 2007, A\&A, 473, 323

Desort, M., Lagrange, A.-M., Galland, F., Udry, S., \& Mayor, M. 2007, A\&A, 473,983

ESA 1997, The Hipparcos and Tycho Catalogues

Fischer, D. A., \& Valenti, J. 2005, ApJ, 622, 1102

Fischer, D. A., Laughlin, G., Butler, P., et al. 2005, ApJ, 620, 481

Fortney, J. J., Marley, M. S., \& Barnes, J. W. 2007, ApJ, 659, 1661

Gonzalez, G. 1998, A\&A, 334, 221

Hekker, S., \& Meléndez, J. 2007, A\&A, 475, 1003

Ida, S., \& Lin, D. N. C. 2004a, ApJ, 604, 388

Ida, S., \& Lin, D. N. C. 2004b, ApJ, 616, 567

Matsuo, T., Shibai, H., Ootsubo, T., \& Tamura, M. 2007, ApJ, 662, 1282

Melo, C., Santos, N. C., Gieren, W., et al. 2007, A\&A, 467, 721

Mordasini, C., Alibert, Y., Benz, W., \& Naef, D. 2007, ArXiv e-prints, 710

Moutou, C., Loeillet, B., Bouchy, F., et al. 2006, A\&A, 458, 327

Pasquini, L., Döllinger, M. P., Weiss, A., et al. 2007, A\&A, 473, 979

Pont, F., Bouchy, F., Melo, C., et al. 2005, A\&A, 438, 1123

Queloz, D., Henry, G. W., Sivan, J. P., et al. 2001, A\&A, 379, 279

Robinson, S. E., Ammons, S. M., Kretke, K. A., et al. 2007, ApJS, 169, 430

Santos, N. C., Mayor, M., Naef, D., et al. 2000, A\&A, 361, 265

Santos, N. C., Mayor, M., Naef, D., et al. 2002, A\&A, 392, 215

Santos, N. C., Udry, S., Mayor, M., et al. 2003, A\&A, 406, 373

Santos, N. C., Israelian, G., \& Mayor, M. 2004, A\&A, 415, 1153

Sato, B., Fischer, D. A., Henry, G. W., et al. 2005, ApJ, 633, 465

Schaerer, D., Charbonnel, C., Meynet, G., Maeder, A., \& Schaller, G. 1993, A\&AS, 102, 339

Strassmeier, K., Washuettl, A., Granzer, T., Scheck, M., \& Weber, M. 2000, A\&AS, 142, 275

Tinney, C. G., Butler, R. P., Marcy, G. W., et al. 2002, ApJ, 571, 528

Udry, S., \& Santos, N. 2007, ARA\&A, 45, 397

Udry, S., Mayor, M., \& Santos, N. 2003, A\&A, 407, 369

Valencia, D., O'Connell, R. J., \& Sasselov, D. 2006, Icarus, 181, 545 